\title{
ON DEHN FUNCTIONS AND PRODUCTS OF GROUPS
}

\author{
STEPHEN G. BRICK
}

\begin{abstract}
If $G$ is a finitely presented group then its Dehn function-or its isoperimetric inequality-is of interest. For example, $G$ satisfies a linear isoperimetric inequality iff $G$ is negatively curved (or hyperbolic in the sense of Gromov). Also, if $G$ possesses an automatic structure then $G$ satisfies a quadratic isoperimetric inequality.

We investigate the effect of certain natural operations on the Dehn function. We consider direct products, taking subgroups of finite index, free products, amalgamations, and HNN extensions.
\end{abstract}

\section{INTRODUCTION}

The study of isoperimetric inequalities for finitely presented groups can be approached in two different ways. There is the geometric approach (see [Gr]). Given a finitely presented group $G$, choose a compact Riemannian manifold $M$ with fundamental group being $G$. Then consider embedded circles which bound disks in $M$, and search for a relationship between the length of the circle and the area of a minimal spanning disk. One can then triangulate $M$ and take simplicial approximations, resulting in immersed disks. What was their area then becomes the number of two-cells in the image of the immersion counted with multiplicity. We are thus led to a combinatorial approach to the isoperimetric inequality (also see [Ge and CEHLPT]).

We start by defining the Dehn function of a finite two-complex. Let $K$ be a finite two-complex. If $w$ is a circuit in $K^{(1)}$, null-homotopic in $K$, then there is a Van Kampen diagram for $w$, i.e. a pair $(D, j)$ where $D$ is a simplyconnected finite subcomplex of the plane and $j: D \rightarrow K$ is a combinatorial map with boundary label being $w$. We will often omit the mention of the map $j$. Given a Van Kampen diagram $D$, let $a(D)$ be the number of faces of the diagram - this is the combinatorial analogue of area-and define

$$
\Delta_{K}(w)=\min \{a(D) \mid D \text { is a Van Kampen diagram for } w\} .
$$

Alternatively, we could look at transverse maps of disks into $K$, with boundary mapping to $w$, and take the minimal number of preimages of two-cells. Define, for $l \in \mathbf{N}, N_{K}(l)$ to be the set of circuits in $K^{(1)}$ that are null-homotopic in $K$ and of length less than or equal to $l$. Then the Dehn function of the complex

Received by the editors October 11, 1990.

1980 Mathematics Subject Classification (1985 Revision). Primary 57M20; Secondary 20F05.

Key words and phrases. Dehn function, isoperimetric inequality, finitely presented group, direct product, free product with amalgamation. 
$K$ is the monotone function $\delta_{K}: \mathbf{N} \rightarrow \mathbf{N}$ (where $\mathbf{N}=\{0,1,2, \ldots\}$ ) given by

$$
\delta_{K}(l)=\max \left\{\Delta_{K}(w) \mid w \in N_{K}(l)\right\} .
$$

It is worth noting that originally Gersten, in an earlier version of [Ge], studied this function, but his current approach is slightly different from ours. Essentially, he uses, what we call in $\S 3$, the subnegative closure of our Dehn function.

Now given a finite presentation of a group $G$,

$$
\mathscr{P}=\left\langle x_{1}, x_{2}, \ldots, x_{n} \mid r_{1}, r_{2}, \ldots, r_{k}\right\rangle,
$$

we define the Dehn functions of the presentation $\mathscr{P}$ in terms of those of the associated two-complex. Algebraically, this may be formulated as follows (as this is the case of interest, we give slightly more detailed definitions).

Let $F_{\mathscr{P}}=F\left(x_{1}, x_{2}, \ldots, x_{n}\right)$ denote the free group on the generators $x_{1}, x_{2}$, $\ldots, x_{n}$ and $R_{\mathscr{P}}=\left\{r_{1}, r_{2}, \ldots, r_{k}, r_{1}^{-1}, \ldots, r_{k}^{-1}\right\}$. Given a word $w$ in the generators $x_{1}, \ldots, x_{n}$ write $|w|_{\mathscr{P}}$ for its length. We set $N_{\mathscr{P}}=\left\langle\left\langle R_{\mathscr{P}}\right\rangle\right\rangle_{F_{\mathscr{P}}}$ the normal closure of the relators in $F_{\mathscr{D}} . N_{\mathscr{D}}$ corresponds to the set of null-homotopic circuits. Define the area function of $\mathscr{P}, \Delta_{\mathscr{D}}: N_{\mathscr{P}} \rightarrow \mathbf{N}=\{0,1,2, \ldots\}$ as follows:

$$
\begin{array}{r}
\Delta_{\mathscr{P}}(w)=\min \left\{m \mid \exists s_{1}, s_{2}, \ldots, s_{m} \in R_{\mathscr{P}} \text { and } \exists g_{1}, g_{2}, \ldots, g_{m} \in F\right. \\
\text { with } \left.w=\prod_{i=1}^{m} g_{i} s_{i} g_{i}^{-1}\right\} .
\end{array}
$$

We will call such a product for $w$ a Dehn product over $\mathscr{P}$ and will call $m$ its area. If the Dehn product has minimal area, call it a minimal Dehn product for $w$ over $\mathscr{P}$. Given $l \in \mathbf{N}$ let $N_{\mathscr{P}}(l)=\left\{\left.w \in N_{\mathscr{P}}|| w\right|_{\mathscr{P}} \leq l\right\}$. Then the Dehn function of the presentation $\mathscr{P}$ is the function $\delta_{\mathscr{P}}: \mathbf{N} \rightarrow \mathbf{N}$ defined by

$$
\delta_{\mathscr{P}}(l)=\max \left\{\Delta_{\mathscr{P}}(w) \mid w \in N_{\mathscr{P}}(l)\right\} .
$$

Clearly this is the same function as $\delta_{K(\mathscr{P})}$ where $K(\mathscr{P})$ is the two-complex associated to the presentation $\mathscr{P}$.

Note that a finite presentation has solvable word problem iff its Dehn function is recursive (see [Ge] or [CEHLPT]).

It turns out that the Dehn functions depend on the finite presentation chosen. However the type of function-for example whether it is linear, quadratic, etc.-does not depend on the presentation (this is made precise in $\S 1$ below). So if $G$ has a finite presentation which in turn has Dehn function that is bounded above by a function that is linear, quadratic, etc., then we will say that $G$ satisfies a linear, quadratic, etc. isoperimetric inequality. Note that, a priori, the Dehn function may be bounded above by, for instance, a quadratic function, but not be a quadratic function itself.

A finitely presented group is said to be negatively curved if it satisfies a linear isoperimetric inequality. Such groups are also called hyperbolic (see [Gr, Ba et al. and CDP]). Some examples of negatively curved groups are finitely generated free groups and fundamental groups of closed hyperbolic manifolds. It is a theorem of Gromov's that $\mathbf{Z} \oplus \mathbf{Z}$ is not embeddable in any negatively curved group. So the group of the trefoil knot, and in fact of any classical knot group, is not negatively curved. But the trefoil knot group can be expressed as a free 
product of two infinite cyclic groups with cyclic amalgamation. Thus we see that the class of negatively curved groups is not closed under free products with amalgamation. However, it is a result of [BGSS] that the free product of two negatively curved groups with cyclic amalgamation possesses an automatic structure. Groups with automatic structures were introduced in [CEHLPT], where among other results, it is proved that such groups satisfy a quadratic isoperimetric inequality (see [BGSS] for another proof). So in particular, the trefoil knot group satisfies a quadratic isoperimetric inequality.

Free products with amalgamation can change the Dehn function in different ways. It remains unchanged when the factors have linear Dehn functions and the amalgamating subgroup is finite (see [Gr]). It can change from linear to quadratic as in the case mentioned above of the trefoil knot group. And it can change from linear to exponential as in a case involving the Baumslag-Solitar groups (see [Ge]).

The layout of our paper is as follows. In $\S 1$, we study the effect of a change of presentation on the Dehn function obtained. The main idea is to see how a Tietze transformation changes the Dehn function. We also show, by looking at finite sheeted covers, that the type of Dehn function is unchanged upon passage to a subgroup of finite index.

In $\S 2$, we consider direct products. We show that the greatest change occurs when one takes a direct product of two negatively curved groups.

In $\S 3$, we look at free products, amalgamations and HNN extensions. We prove that the Dehn function of a free product-or an amalgamation or HNN extension along a finite group-is bounded above by the subnegative closures of the Dehn functions of the factors. We also consider amalgamations where the subgroup is plus-isometric, in the sense of Gromov, in each of the factors. In this case we show that the Dehn function may be changed by multiplying by a linear term.

Additional results on Dehn functions can be found in [A and $\mathrm{Br}$ ].

\section{DeHN FUNCTIONS, PRELIMINARY RESUltS}

Different presentations can yield different Dehn functions. Consider, for example, the following two presentations of $\mathbf{Z}$ :

$$
\mathscr{P}_{1}=\langle x \mid\rangle, \quad \mathscr{P}_{2}=\langle x, y \mid y\rangle .
$$

As $\mathscr{P}_{1}$ has no relators, $\delta_{\mathscr{P}_{1}}$ is identically zero. However, the same is not true for $\delta_{\mathscr{R}_{2}}$. Consider the word $w=y^{l}$. It is immediate that $w \in N_{\mathscr{P}_{2}}(l)$ and $\Delta_{\mathscr{P}_{2}}(w)=l$. So $\delta_{\mathscr{P}_{2}}(l) \geq l$. In fact one can easily see that equality holds. Passage from $\mathscr{P}_{1}$ to $\mathscr{P}_{2}$ is a particular type of Tietze transformation and has a particular effect on the Dehn function. Other Tietze transformations result in other effects. A study of this nature tells us how Dehn functions change when the presentation is changed. Specifically, we have the following (also see [Ge] or [CEHLPT]):

Proposition 1.1. If $\mathscr{P}_{1}$ and $\mathscr{P}_{2}$ are finite presentations of the same group then there are constants $A, B$, and $C$ such that

$$
\delta_{\mathscr{P}_{2}}(l) \leq A \cdot l+B \cdot \delta_{\mathscr{P}_{1}}(C \cdot l)
$$

for all $l \in \mathbf{N}$. (Note-it also follows that $\delta_{\mathscr{D}_{1}}$ can be bounded above by a similar expression involving $\delta_{\mathscr{P}_{2}}$.) 
Proof. One can obtain $\mathscr{P}_{2}$ from $\mathscr{P}_{1}$ by an application of a finite sequence of Tietze transformations. Let us prove the result when $\mathscr{P}_{2}$ is gotten from $\mathscr{P}_{1}$ by applying a single Tietze transformation. We consider the different types of Tietz transformations separately. Since the desired relation is clearly transitive, this suffices.

Consider the different possible Tietze transformations (for simplicity sake, we use a slightly longer than usual list):

(T0) A free reduction of a relator.

(T1) Replace some relator $r$ by $r^{-1}$.

(T2) Replace some relator $r$ by $g_{r} g^{-1}$ where $g \in F_{\mathscr{P}_{1}}$.

(T3) Replace some pair of relators $\left(r, r^{\prime}\right)$ by $\left(r, r r^{\prime}\right)$.

(T4) Add a new generator $x$ and a new relator $x u^{-1}$ where $u \in F_{\mathscr{P}_{1}}$ (so $u$ does not involve $x$ ).

(T5) The inverse transformation to (T4).

(T6) Add a trivial relator " 1 ".

(T7) The inverse transformation to (T6).

Consider the transformations (T0), (T1), (T2), (T6), and (T7). It is clear that $F_{\mathscr{P}}, N_{\mathscr{P}}$, and $N_{\mathscr{P}}(l)$ are unchanged. In the case of (T0), (T1), or (T2), any Dehn product over $\mathscr{P}_{1}$ can be easily transformed to a Dehn product over $\mathscr{P}_{2}$ of the same area (for instance, for (T2), replace any $g_{i} r g_{i}^{-1}$ by $\left(g_{i} g^{-1}\right)\left(g r g^{-1}\right)\left(g_{i} g^{-1}\right)^{-1}$; similarly if $g_{i} r^{-1} g_{i}^{-1}$ occurs in the Dehn product). For either (T6) or (T7) it suffices to observe that a minimal Dehn product never contains a trivial relator. Hence the Dehn function is unchanged for these transformations.

In the case of (T3), $F_{\mathscr{P}}, N_{\mathscr{P}}$, and $N_{\mathscr{P}}(l)$ are also unchanged. However, transforming a Dehn product over $\mathscr{P}_{1}$ into a Dehn product over $\mathscr{P}_{2}$ may increase the area. One replaces each occurrence of $g_{i} r^{\prime} g_{i}^{-1}$ by the product $\left(g_{i} r^{-1} g_{i}^{-1}\right)\left(g_{i} r r^{\prime} g_{i}^{-1}\right)$, and one replaces occurrences of $g_{i} r^{-1} g_{i}^{-1}$ by $\left(g_{i}\left(r r^{\prime}\right)^{-1} g_{i}^{-1}\right)\left(g_{i} r g_{i}^{-1}\right)$. Thus one obtains $\Delta_{\mathscr{P}_{2}}(l) \leq 2 \delta_{\mathscr{P}_{1}}(l)$. Taking the maximum over $N_{\mathscr{P}_{2}}(l)$ yields the result $\delta_{\mathscr{P}_{2}} \leq 2 \delta_{\mathscr{P}_{1}}$.

As the arguments for the transformation (T4) and its inverse (T5) are slightly more complicated, we give more details. Denote by $\rho$ the new relator $x u^{-1}$. Observe that $F_{\mathscr{P}}$ and $N_{\mathscr{P}}(l)$ are different for the two presentations. However, $F_{\mathscr{P}_{1}} \subset F_{\mathscr{P}_{2}}$ and there is a retraction $\alpha: F_{\mathscr{P}_{2}} \rightarrow F_{\mathscr{P}_{1}}$ defined on the new generator by $x \mapsto u$. This map induces the identity on the group of the presentations. Note that $\alpha$ may increase the length of a word. Let $C$ be the length of $u$ in $F_{\mathscr{P}_{1}}$. If $v \in F_{\mathscr{P}_{2}}$ and $|v|_{\mathscr{P}_{2}} \leq l$ then $|\alpha(v)|_{\mathscr{P}_{1}} \leq C \cdot l$. Moreover $\alpha\left(R_{\mathscr{P}_{2}}\right)=$ $R_{\mathscr{P}_{1}} \cup\left\{u^{-1} \cdot u\right\} \subset N_{\mathscr{P}_{1}}$. From this we see that $\alpha\left(N_{\mathscr{P}_{2}}(l)\right) \subset N_{\mathscr{P}_{1}}(C \cdot l)$.

Fix an integer $l$. Choose $w \in N_{\mathscr{P}_{2}}(l)$ with $\delta_{\mathscr{P}_{2}}(l)=\Delta_{\mathscr{P}_{2}}(w)$. Now $\alpha(w) \in$ $N_{\mathscr{P}_{1}}(C \cdot l)$. Assume that $m=\Delta_{\mathscr{P}_{1}}(\alpha(w))$ and express $\alpha(w)$ as a product:

$$
\alpha(w)=\prod_{i=1}^{m} g_{i} s_{i} g_{i}^{-1}
$$

where $s_{1}, s_{2}, \ldots, s_{m} \in R_{\mathscr{P}_{1}}, g_{1}, g_{2}, \ldots, g_{m} \in F_{\mathscr{P}_{1}} \subset F_{\mathscr{P}_{2}}$. But $w \cdot(\alpha(w))^{-1}$ $\in N_{\mathscr{R}_{2}}$ and can be written as a product of $l^{\prime}$ conjugates of the relator $\rho$ or its inverse, where $l^{\prime}$ is the number of occurrences of $x$ or $x^{-1}$ in $w$ (see 


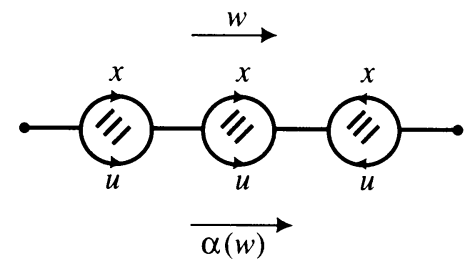

FIGURE 1.1

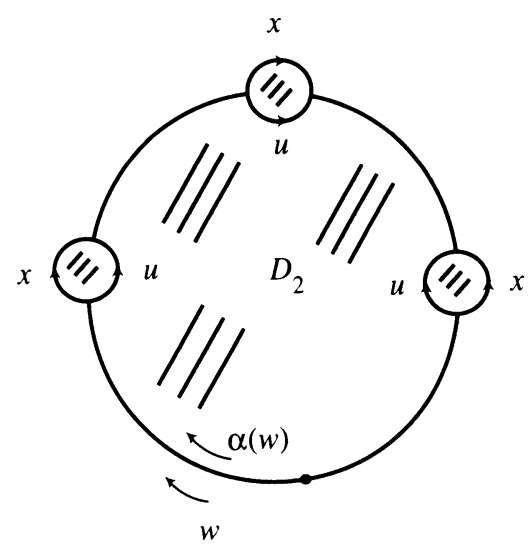

FIGURE 1.2

Figure 1.1 for a Van Kampen diagram). Further, $R_{\mathscr{P}_{1}} \subset R_{\mathscr{P}_{2}}$ and

$$
w=\left(w \cdot(\alpha(w))^{-1}\right) \cdot(\alpha(w))=\left(l^{\prime} \text { conjugates }\right) \cdot(m \text { conjugates }) .
$$

One can view this as glueing the Van Kampen diagram $D_{1}$ for $w \alpha(w)^{-1}$ to the diagram $D_{2}$ for $\alpha(w)$ along the path mapping to $\alpha(w)$ (see Figure 1.2). It follows that $\Delta_{\mathscr{P}_{2}}(w) \leq l^{\prime}+m$. Since $m=\Delta_{\mathscr{P}_{1}}(\alpha(w))$ and $\alpha(w) \in N_{\mathscr{P}_{1}}(C \cdot l)$ we have $m \leq \delta_{\mathscr{P}_{1}}(C \cdot l)$. Also $l^{\prime} \leq l$ and $\delta_{\mathscr{P}_{2}}(l)=\Delta \mathscr{\mathscr { P }}_{2}(w)$. Hence $\delta_{\mathscr{P}_{2}}(l) \leq$ $l+\delta_{\mathscr{P}_{1}}(C \cdot l)$.

On the other hand, consider the inverse transformation (T5) from $\mathscr{P}_{2}$ to $\mathscr{P}_{1}$. Now $F_{\mathscr{P}_{1}} \subset F_{\mathscr{P}_{2}}, R_{\mathscr{P}_{1}} \subset R_{\mathscr{P}_{2}}$ and the inclusion map does not change the length of a word. It follows for any $w \in N_{\mathscr{P}_{1}}(l)$ that $\Delta_{\mathscr{P}_{2}}(w) \leq \Delta_{\mathscr{P}_{1}}(w)$ and, as $N_{\mathscr{P}_{1}}(l) \subset N_{\mathscr{P}_{2}}(l)$, we get, after taking maximums, that $\delta_{\mathscr{P}_{1}}(l) \leq \delta_{\mathscr{P}_{2}}(l)$.

From the preceding proposition, we see that, though a change of presentation may change the Dehn functions, the "type" of functions remains unchanged. In order to make this precise, we introduce a few definitions. Given two functions $f, g \in \mathbf{N} \rightarrow \mathbf{N}$, we say that $f \preccurlyeq g$ (also written $f(l) \preccurlyeq g(l)$ if we need to stress that $f$ and $g$ are functions of $l$ ) if there are constants $A, B$, and $C$ such that

$$
f(l) \leq A \cdot l+B \cdot g(C \cdot l)
$$

for all $l \in \mathbf{N}$. We say that $f$ and $g$ are of the same type, and write $f \equiv g$, if $f \preccurlyeq g$ and $g \preccurlyeq f$. Note this is an equivalence relation. We will write $[f]$ for the $\equiv$-class of $f$. Observe that if $f \equiv g$ then $f$ and $g$ have the same growth.

Multiplication and addition respect this equivalence relation. Further, the relation $\preccurlyeq$ induces a relation, likewise denoted $\preccurlyeq$, on the $\equiv$-classes. Also, we will find it useful to write $\max ([f],[g])$ for $[\max (f, g)]$.

Using this definition, the conclusion in Proposition 1.1 becomes " $\delta_{\mathscr{P}_{1}}$ and $\delta_{\mathscr{P}_{2}}$ are of the same type." With this is mind, we say that a function $f: \mathbf{N} \rightarrow \mathbf{N}$ is a Dehn function for a finitely presented group $G$ if $f \equiv g$ where $g$ is a Dehn function of some finite presentation $\mathscr{P}$. We write $\delta_{G}$ for the $\equiv$-equivalence class $\left[\delta_{\mathscr{P}}\right]$ where $\mathscr{P}$ is some finite presentation of $G$. We will call $\delta_{G}$ the Dehn function of $G$.

Of special interest is the case when $\delta_{G} \preccurlyeq\left[l^{n}\right]$. To stress this case, we associate a degree to a finitely presented group $G, \operatorname{deg}(G)$, called the Dehn degree of $G$, by letting $\operatorname{deg}(G) \leq n$ if $\delta_{G} \preccurlyeq\left[l^{n}\right]$. So a group $G$ is negatively curved if 
$\operatorname{deg}(G)=1$. And if $G$ possesses an automatic structure then $\operatorname{deg}(G) \leq 2$. Note that there are groups-such as the Baumslag-Solitar group-of infinite Dehn degree (see [Ge]).

We turn now to the Dehn functions of finite connected two-complexes. Our aim is to show that the type of function only depends on the fundamental group. We start with a lemma.

Lemma 1.2. Suppose $K$ is a finite connected two-complex. Let $T$ be a maximal tree in $K^{(1)}$ and set $L=K / T$ and let $\mathscr{P}$ be the finite presentation associated to $L$. Then $\delta_{K} \equiv \delta_{\mathscr{P}}$.

Proof. Let $\alpha: K \rightarrow L=K / T$ be the natural map. Since $T$ is a finite tree there is a bound $c$ to the length of a reduced path in $T$. Let $C=2 c+1$. Suppose that $w \in N_{\mathscr{P}}(l)$ with $\delta_{\mathscr{P}}(l)=\Delta_{\mathscr{P}}(w)$. We may view $w$ as a circuit in $L^{(1)}$ that is null-homotopic in $L$. Every edge of $L$ lifts to a unique edge of $K \backslash T$. Do this for each of the edges occurring in the circuit $w$. The result is not a circuit in $K$, but it can be made into a circuit in $K$ by joining the endpoints of the lifted edges by reduced paths in $T$. This gives rise to a circuit in $K$ of length bounded by $C \cdot l$. Denote it by $\omega$. Clearly, since $\alpha$ collapses $T$ to a point, $\alpha(\omega)=w$. Also, the Van-Kampen diagram for $w$ lifts to one for $\omega$. Thus $\omega \in N_{K}(C \cdot l)$. Hence, there is a Van Kampen diagram $(D, j)$ for $\omega$ with $a(D) \leq \delta_{K}(C \cdot l)$. Now project the diagram down via $\alpha$ yielding a Van Kampen diagram $(D, \alpha \circ j)$ for $\alpha(\omega)=w$. We write $\alpha(D)$ for this diagram. Then

$$
a(\alpha(D))=a(D) \leq \delta_{K}(C \cdot l)
$$

and it follows that $\Delta_{\mathscr{P}}(w) \leq \delta_{K}(C \cdot l)$. Since $w$ was chosen with $\delta_{\mathscr{P}}(l)=$ $\Delta_{\mathscr{P}}(w)$, we have that $\delta_{\mathscr{P}} \preccurlyeq \delta_{K}$.

Now suppose $w^{\prime}$ is a circuit in $K^{(1)}$ null-homotopic in $K$ of length less than or equal to $l$. Consider $\alpha\left(w^{\prime}\right)$. This is a null-homotopic loop in $L$ of possibly shorter length. In any case, $\alpha\left(w^{\prime}\right)$ can be thought of as an element of $N_{\mathscr{P}}(l)$. It can then be expressed as a product of no more than $\delta_{\mathscr{D}}(l)$ conjugates of relators or their inverses. Each relator comes from some two-cell of $K$. Thus, one gets a Van Kampen diagram $D$ for $w^{\prime}$ with $a(D) \leq \delta_{\mathscr{P}}(l)$. It follows that $\Delta_{K}\left(w^{\prime}\right) \leq \delta_{\mathscr{P}}(l)$. This is true for any $w^{\prime} \in N_{K}(l)$. Hence $\delta_{K}(l) \leq \delta_{\mathscr{P}}(l)$. And we get that $\delta_{K} \preccurlyeq \delta_{\mathscr{P}}$.

Applying the previous two results yields the following corollary (also see [Ge]).

Corollary 1.3. Let $K_{1}$ and $K_{2}$ be finite connected two-complexes with isomorphic fundamental groups. Then $\delta_{K_{1}} \equiv \delta_{K_{2}}$.

Proof. Let $T_{1}$ and $T_{2}$ be maximal trees in $K_{1}^{(1)}$ and $K_{2}^{(1)}$ respectively. Let $\mathscr{P}_{1}$ and $\mathscr{P}_{2}$ be the corresponding finite presentations. By hypothesis, $\mathscr{P}_{1}$ and $\mathscr{P}_{2}$ are presentations of the same group. Hence, by Proposition 1.1, $\delta_{\mathscr{P}_{1}} \equiv \delta_{\mathscr{P}_{2}}$. By Lemma $1.2, \delta_{K_{1}} \equiv \delta_{\mathscr{P}_{1}}$ and $\delta_{K_{2}} \equiv \delta_{\mathscr{P}_{2}}$. Transitivity now gives our result.

So in particular the Dehn function of a finite two-complex only depends on the fundamental group (and not, for instance, on the cell structure). Also, it follows that to compute the Dehn function of a group $G$ it suffices to look at any finite connected two-complex with fundamental group $G$. 
It is worth noting that the notion of a Dehn function easily extends to infinite two-complexes. The only changes are that $N_{K}(l)$ may be infinite, we have to take the supremum instead of the maximum, and the result may take on the value $+\infty$. Using this extended definition, it is easy to prove

Proposition 1.4. Let $p: L \rightarrow K$ be a covering space. Then $\delta_{L}=\delta_{K}$.

Proof. Given a circuit $w \in N_{K}(l)$, lift $w$ to a circuit $w^{\prime}$ (it lifts since it is null-homotopic). We then have $w^{\prime} \in N_{L}(l)$. So we can find a Van Kampen diagram $(D, j)$ for $w^{\prime}$ with $a(D) \leq \delta_{L}(l)$. But then $p(D)=(D, p \circ j)$ is a Van Kampen diagram for $w$ with $a(p(D))=a(D)$. Then $\Delta_{K}(w) \leq a(D) \leq \delta_{L}(l)$. Taking the supremum over $N_{K}(l)$ yields $\delta_{K}(l) \leq \delta_{L}(l)$.

Now suppose $w^{\prime} \in N_{L}(l)$. Project down via $p$. Clearly we have that $p\left(w^{\prime}\right) \in$ $N_{K}(l)$. Take a Van Kampen diagram $D$ for $p\left(w^{\prime}\right)$ with $a(D)=\Delta_{K}\left(p\left(w^{\prime}\right)\right) \leq$ $\delta_{K}(l)$. This diagram lifts to one for $w^{\prime}$ having the same area. Thus $\Delta_{L}\left(w^{\prime}\right) \leq$ $a(D) \leq \delta_{K}(l)$. Now take the supremum over $N_{L}(l)$, giving $\delta_{L}(l) \leq \delta_{K}(l)$.

One consequence of this result is that the Dehn function of an infinite complex depends on the cell structure. For instance, the plane with one cell structure covers the torus, and with another covers the closed hyperbolic surface of genus two. In the first case the Dehn function is quadratic (as the fundamental group of the torus is $\mathbf{Z} \oplus \mathbf{Z}$ ), while in the second case the Dehn function is linear (see [Gr]).

However, we can use Proposition 1.4 to obtain the following result:

Corollary 1.5. Let $G$ be a finitely presented group and $H$ a subgroup of finite index. Then $\delta_{H} \equiv \delta_{G}$.

Proof. Let $K$ be a finite two-complex with fundamental group $G$. The covering space corresponding to $H$ is finite-sheeted, and so is a finite complex. Hence, it may be used to compute the Dehn function of $H$. Our result now follows from Proposition 1.4.

\section{DiRECT PRODUCTS}

We turn to the effect of taking a direct product (or sum) on the Dehn function. As seen above, the integers, $\mathbf{Z}$, satisfy a linear isoperimetric inequality. It is well known that $\mathbf{Z} \oplus \mathbf{Z}$ satisfies a quadratic isoperimetric inequality. So a direct product may change the Dehn function from linear to quadratic. In fact, in a sense, that is the full extent of the possible change.

Proposition 2.1. Let $G$ and $H$ be finitely presented groups. Then

$$
\delta_{G \oplus H} \preccurlyeq a \text { quadratic function }+\delta_{G}+\delta_{H} .
$$

Note: $\delta_{G}+\delta_{H}$ could be replaced by $\max \left(\delta_{G}, \delta_{H}\right)$ as they are of the same type. Proof. Let

$$
\mathscr{P}_{G}=\left\langle x_{1}, x_{2}, \ldots, x_{n} \mid r_{1}, r_{2}, \ldots, r_{k}\right\rangle
$$

be a presentation for $G$ and

$$
\mathscr{P}_{H}=\left\langle y_{1}, y_{2}, \ldots, y_{m} \mid s_{1}, s_{2}, \ldots, s_{t}\right\rangle
$$




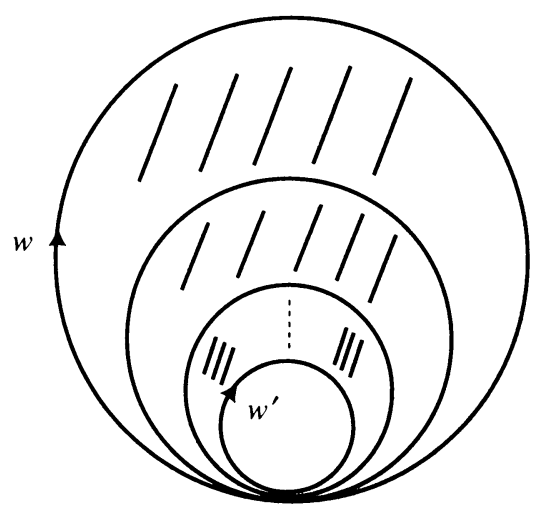

FIGURE 2.1

be a presentation for $H$. Consider

$$
\mathscr{P}=\left\langle x_{i}, y_{j} \mid r_{p}, s_{q},\left[x_{i}, y_{j}\right]\right\rangle,
$$

a presentation for $G \oplus H$. Given $w \in N_{\mathscr{P}}(l)$ with length $|w|_{\mathscr{P}} \leq l$, we can "homotop" $w$ to a word of the form $w^{\prime}=U \cdot V$ where $U$ is a word in the $x_{i}$ 's only, and $V$ a word in the $y_{i}$ 's only. One does this a letter at a time. So we have a number of singular annuli $A_{i}$ glued together, resulting in a singular annulus $A$ with outer boundary components $w$ and inner boundary component $w^{\prime}$. (See Figure 2.1.) Here each $A_{i}$ has area less than $|w|_{\mathscr{P}} \leq l$. Moreover the number $h$ of subannuli is equal to the number of occurrences of some $x$ after some $y_{j}^{ \pm 1}$. Thus $h \leq|w|_{\mathscr{P}} \leq l$ and so the area of $A$ is bounded above by $l^{2}$.

It remains to find the area of some Van Kampen diagram for $w^{\prime}$.

It is easy to see that $U\left(x_{i}\right)$ and $\left(V\left(y_{j}\right)\right)^{-1}$ represent the identity. As their lengths are bounded above by $l$, we can find Van Kampen diagrams $D_{U}$ and $D_{V}$ with $a\left(D_{U}\right) \leq \delta_{\mathscr{P}_{G}}(l)$ and $a\left(D_{V}\right) \leq \delta_{\mathscr{P}_{H}}(l)$. (Formally, these are diagrams over the presentations $\mathscr{P}_{G}$ and $\mathscr{P}_{H}$. But, since these are both subpresentations of $\mathscr{P}$, we can consider these as diagrams over $\mathscr{P}$.) Now glue these diagrams together yielding a diagram $D$ for $w^{\prime}$ with area $\leq \delta_{\mathscr{P}_{G}}(l)+\delta_{\mathscr{P}_{H}}(l)$. Combining the diagram $D$ and the annular diagram $A$ yields a diagram for $w$ with area

$$
\leq l^{2}+\delta_{\mathscr{P}_{G}}(l)+\delta_{\mathscr{P}_{H}}(l) .
$$

Taking the maximum over $w \in N_{\mathscr{P}}(l)$ yields the result.

One property that a direct sum, $A \oplus B$, has is that there are retractions $A \oplus B \rightarrow A$ and $A \oplus B \rightarrow B$. And these retractions are induced on the free group level by retractions that respect the relators. This fact will be useful in computing a lower bound for $\delta_{A \oplus B}$. We treat this as a lemma. We need a definition. Given a presentation $\mathscr{P}=\langle X \mid R\rangle$, where $X$ and $R$ are sets of generators and relators respectively, a retract of $\mathscr{P}$ is a subpresentation $\mathscr{P}$, $\mathscr{P}^{\prime}=\left\langle X^{\prime} \mid R^{\prime}\right\rangle$, with $X^{\prime} \subset X$ and $R^{\prime} \subset R$ and with the natural retraction $f: F_{X} \rightarrow F_{X^{\prime}}$ mapping $R \backslash R^{\prime}$ to the identity element of $F_{X^{\prime}}$.

Lemma 2.2. If $\mathscr{P}^{\prime}=\left\langle X^{\prime} \mid R^{\prime}\right\rangle$ is a retract of a finite presentation $\mathscr{P}=\langle X \mid R\rangle$ then $\delta_{\mathscr{P} \prime} \leq \delta_{P}$.

Proof. Clearly, for any integral $l$, we have $N_{\mathscr{P}}(l) \subset N_{\mathscr{P}}(l)$. As

$$
\delta_{\mathscr{P}^{\prime}}(l)=\max \left\{\Delta_{\mathscr{P}^{\prime}}(w) \mid w \in N_{\mathscr{P}^{\prime}}(l)\right\}
$$


and

$$
\delta_{\mathscr{P}}(l)=\max \left\{\Delta_{\mathscr{P}}(v) \mid v \in N_{\mathscr{P}}(l)\right\}
$$

it suffices to show, for $w \in N_{\mathscr{P}}(l)$, that $\Delta_{\mathscr{P} \prime}(w) \leq \Delta_{\mathscr{P}}(w)$.

So, suppose we have a minimal Dehn product for $w$ over $\mathscr{P}$ :

$$
w=\prod_{i}^{m} g_{i} s_{i} g_{i}^{-1} \quad \text { with } g_{i} \in F_{X} \text { and } s_{i} \text { or } s_{i}^{-1} \in R \text {. }
$$

Apply the retraction $f: F_{X} \rightarrow F_{X^{\prime}}$, yielding

$$
w=\prod_{i}^{m} f\left(g_{i}\right) f\left(s_{i}\right) f\left(g_{i}\right)^{-1}
$$

Omitting from the product those terms $f\left(g_{i}\right) f\left(s_{i}\right) f\left(g_{i}\right)^{-1}$ with $f\left(s_{i}\right)=1$ in $F_{X^{\prime}}$, and using the fact that $f$ is a retraction, yields a Dehn product for $w$ over $\mathscr{P}^{\prime}$ of area $\leq m$. Thus $\Delta_{\mathscr{P}}(w) \leq \Delta_{\mathscr{P}}(w)$.

Using the above, we obtain

Corollary 2.3. Let $G$ and $H$ be finitely presented groups. Then $\max \left(\delta_{G}, \delta_{H}\right) \preccurlyeq$ $\delta_{G \oplus H}$.

Proof. As above, let

$$
\mathscr{P}_{G}=\left\langle x_{1}, x_{2}, \ldots, x_{n} \mid r_{1}, r_{2}, \ldots, r_{k}\right\rangle
$$

be a presentation for $G$ and

$$
\mathscr{P}_{H}=\left\langle y_{1}, y_{2}, \ldots, y_{m} \mid s_{1}, s_{2}, \ldots, s_{t}\right\rangle
$$

be a presentation for $H$. Take, as presentation for $G \oplus H$ :

$$
\mathscr{P}=\left\langle x_{i}, y_{j} \mid r_{p}, s_{q},\left[x_{i}, y_{j}\right]\right\rangle \text {. }
$$

Clearly $\mathscr{P}_{H}$ and $\mathscr{P}_{G}$ are both retracts of $\mathscr{P}$. Hence the result follows from Lemma 2.2.

Combining Corollary 2.3 and Proposition 2.1 yields

Corollary 2.4. Let $G$ and $H$ be finitely presented groups; then

$$
\max (\operatorname{deg}(G), \operatorname{deg}(H)) \leq \operatorname{deg}(G \oplus H) \leq \max (\operatorname{deg}(G), \operatorname{deg}(H), 2) .
$$

Using a result of Gromov's together with a result of Short's, we get

Corollary 2.5. Let $G$ and $H$ be finitely presented infinite groups. Then

$$
\operatorname{deg}(G \oplus H)=\max (\operatorname{deg}(G), \operatorname{deg}(H), 2) .
$$

Proof. By Corollary 2.4, we need only show that if both $G$ and $H$ are negatively curved then $G \oplus H$ is not negatively curved. But then, by [Sh] both $G$ and $H$ contain some element of infinite order, and so $G \oplus H$ contains $\mathbf{Z} \oplus \mathbf{Z}$ as a subgroup. Hence, by [Gr], $G \oplus H$ cannot be negatively curved.

\section{Free products AND AMAlgamations}

In finding an upper bound of the Dehn function of a direct product, we used homotopies to change a given word into one of specific form-namely one of the form $U\left(x_{i}\right) \cdot V\left(y_{j}\right)$. We then showed that both $U$ and $V$ represented the 
trivial element. This will be adapted for free products, the difference being that we may have more than two subwords, and we can only conclude that one of the subwords represents the trivial element. But this is sufficient for our purposes. However, there is a technical detail that we must address. For this we need a few definitions.

Let $f: \mathbf{N} \rightarrow \mathbf{N}$ be a function. We say that $f$ is subnegative if for all $l_{1}$ and $l_{2}$ we have that $f\left(l_{1}\right)+f\left(l_{2}\right) \leq f\left(l_{1}+l_{2}\right)$. For example, any power $f(l)=l^{n}$ is subnegative.

Given any function $h: \mathbf{N} \rightarrow \mathbf{N}$ there is a least subnegative function greater than or equal to $h$. Denote it by $\bar{h}$ and call it the subnegative closure of $h$. Clearly, we have the formula

$$
\bar{h}(k)=\max \left\{\sum_{i=1}^{n} h\left(k_{i}\right) \mid \text { where } \sum_{i=1}^{n} k_{i}=k\right\} .
$$

Observe that if $h_{1} \preccurlyeq h_{2}$ then $\bar{h}_{1} \preccurlyeq \bar{h}_{2}$. So we may take the subnegative closure of 三-classes, that is $[\bar{h}]=[\bar{h}]$. Also, if $h(l) \preccurlyeq l^{n}$ then $\bar{h}(l) \preccurlyeq l^{n}$. And so we may look at the subnegative closure of the Dehn function in order to compute the Dehn degree. (It is perhaps worth noting that it is not true that each $\equiv$-class contains a subnegative function. ${ }^{1}$ )

We start with free products.

Proposition 3.1. If $A$ and $B$ are finitely presented then

$$
\max \left(\delta_{A}, \delta_{B}\right) \preccurlyeq \delta_{A * B} \preccurlyeq \max \left(\bar{\delta}_{A}, \bar{\delta}_{B}\right) .
$$

Proof. Choose a finite presentation

$$
\mathscr{P}_{\mathscr{A}}=\left\langle x_{1}, x_{2}, \ldots, x_{n} \mid r_{1}, r_{2}, \ldots, r_{k}\right\rangle
$$

for $A$, and a finite presentation

$$
\mathscr{P}_{B}=\left\langle y_{1}, y_{2}, \ldots, y_{m} \mid s_{1}, s_{2}, \ldots, s_{t}\right\rangle
$$

for $B$. Take, as presentation of $A * B, \mathscr{P}=\left\langle x_{i}, y_{j} \mid r_{p}, s_{q}\right\rangle$. Observe that $\mathscr{P}_{A}$ and $\mathscr{P}_{B}$ are both retracts of $\mathscr{P}$. Hence, by Lemma $2.2, \max \left(\delta_{\mathscr{P}_{A}}, \delta_{\mathscr{P}_{B}}\right) \leq \delta_{\mathscr{P}}$ giving us the left-hand inequality. We will show that $\delta_{\mathscr{P}} \leq \max \left(\bar{\delta}_{\mathscr{P}_{A}}, \bar{\delta}_{\mathscr{D}_{B}}\right)$. This suffices.

We write $h(l)=\max \left(\bar{\delta}_{\mathscr{P}_{A}}(l), \bar{\delta}_{\mathscr{P}_{B}}(l)\right)$. Note that $h$ is subnegative. Suppose $w \in N_{\mathscr{P}}(l)$. Write $w=w_{1} \cdot w_{2} \cdots w_{L}$ where each subword is a word only in either the $x_{i}$ 's or the $y_{j}$ 's, and successive subwords alternate between the two. Call $L$ the free product length of $w$. We will prove, by induction on $L$, that $\Delta_{\mathscr{P}}(w) \leq h\left(|w|_{\mathscr{P}}\right)$.

If $L=1$ then $w$ can be considered a word in one of the presentations $\mathscr{P}_{A}$ or $\mathscr{P}_{B}$. Without loss of generality, suppose $w$ is a word in the presentation $\mathscr{P}_{A}$. Note that $w$ has the same length when considered as a word in $\mathscr{P}_{A}$. Also, since the inclusion of the subpresentation $\mathscr{P}_{A}$ into $\mathscr{P}$ induces an injection on the group level, we may conclude that $w \in N_{\mathscr{P}_{A}}\left(|w|_{\mathscr{P}}\right)$. Thus there is a Dehn product over $\mathscr{P}_{A}$ for $w$ having area bounded above by $\delta_{\mathscr{P}_{A}}\left(|w|_{\mathscr{P}}\right) \leq h\left(|w|_{\mathscr{P}}\right)$. Since $\mathscr{P}_{A}$ is a subpresentation of $\mathscr{P}$, this product is also a Dehn product over $\mathscr{P}$. Hence $\Delta_{\mathscr{P}}(w) \leq h\left(|w|_{\mathscr{P}}\right)$ in the initial case of $L=1$.

\footnotetext{
${ }^{1}$ This was shown to us by colleagues on the Usenet newsgroup sci.math.
} 


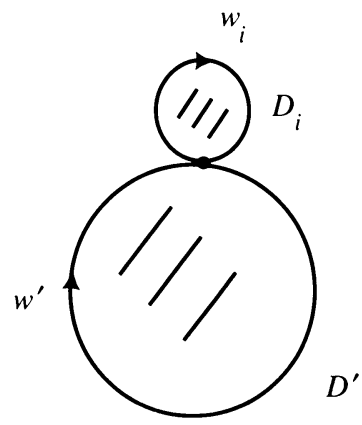

FIGURE 3.1

Now suppose that the result is true for words of free product length less than $L$. Since $w$ represents the identity in $A * B$, at least one of the subwords, say $w_{i}$, represents the identity. We can, as above, consider $w_{i}$ as a word in either $\mathscr{P}_{A}$ or $\mathscr{P}_{B}$ and find a Dehn product over $\mathscr{P}_{A}$ or $\mathscr{P}_{B}$ - which is also a Dehn product over $\mathscr{P}$-of area bounded above by $h\left(\left|w_{i}\right| \mathscr{P}\right)$. Now consider the word $w^{\prime}$ gotten from $w$ by deleting $w_{i}$ :

$$
w^{\prime}=w_{1} \cdots w_{i-1} \cdot w_{i+1} \cdots w_{L}
$$

or possibly $w_{2} \cdots w_{L}$ or $w_{1} \cdots w_{L-1}$. In any case, $w^{\prime}$ has free product length strictly less than $L$ (and $\left.|w|_{\mathscr{D}}=\left|w_{i}\right|_{\mathscr{P}}+\left|w^{\prime}\right|_{\mathscr{D}}\right)$. So there is a Dehn product over $\mathscr{P}$ for $w^{\prime}$ having area bounded above by $h\left(\left|w^{\prime}\right| \mathscr{P}\right)$. Consider the Van Kampen diagrams $D_{i}$ and $D^{\prime}$ that correspond to the Dehn product for $w_{i}$ and $w^{\prime}$. Glue them together (see Figure 3.1) yielding a diagram for $w$ having area bounded above by

$$
h\left(\left|w_{i}\right|_{\mathscr{P}}\right)+h\left(\left|w^{\prime}\right|_{\mathscr{P}}\right) \leq h\left(\left|w_{i}\right|_{\mathscr{P}}+\left|w^{\prime}\right|_{\mathscr{P}}\right)=h\left(|w|_{\mathscr{P}}\right)
$$

by subnegativity of $h$. So, by induction, $\Delta_{\mathscr{P}}(w) \leq h\left(|w|_{\mathscr{P}}\right)$. Taking the maximum over $N_{\mathscr{P}}(l)$ yields the result that $\delta_{\mathscr{P}}(l) \leq h(l)$ as desired.

The argument given above can be altered slightly to obtain the following result (which generalizes the result of Gromov's above negatively curved groups; see [Gr]):

Proposition 3.2. Suppose $A$ and $B$ are finitely presented and $C$ is finite. Let $G=A *_{C} B$. Then $\delta_{G} \preccurlyeq \max \left(\bar{\delta}_{A}, \bar{\delta}_{B}\right)$.

Proof. Enumerate the elements of $C$ by $1, c_{1}, c_{2}, \ldots, c_{d}$. Choose a finite presentation for $A$ of the form

$$
\mathscr{P}_{A}=\left\langle x_{1}, x_{2}, \ldots, x_{n}, c_{1}, c_{2}, \ldots, c_{d} \mid r_{1}, r_{2}, \ldots, r_{u}\right\rangle
$$

and a finite presentation for $B$ of the form

$$
\mathscr{P}_{B}=\left\langle y_{1}, y_{2}, \ldots, y_{m}, c_{1}, c_{2}, \ldots, c_{d} \mid s_{1}, s_{2}, \ldots, s_{t}\right\rangle .
$$

Take, as presentation of $A *_{C} B, \mathscr{P}=\left\langle x_{i}, y_{j}, c_{k} \mid r_{p}, s_{q}\right\rangle$. Write $h(l)=$ $\max \left(\bar{\delta}_{\mathscr{P}_{A}}(l), \bar{\delta}_{\mathscr{P}_{B}}(l)\right)$, which is subnegative. We will show that $\delta_{\mathscr{D}}(l) \leq h(2 \cdot l)$.

Given $w \in N_{\mathscr{P}}(l)$, write $w=w_{1} \cdot w_{2} \cdots w_{L}$ where each subword involves some $x_{i}$ or some $y_{j}$ and successive subwords alternate between the $x_{i}$ 's and the $y_{j}$ 's. However a subword may involve some of the $c_{k}$ 's. We treat $w$ as a cyclic word, and set $L_{w}$ to be the shortest free product length among all of 
the cyclic conjugates of $w$. We choose a cyclic conjugate having free product length equal to this cyclic free product length of $w$. The point is that any Van Kampen diagram for $w$ is also a Van Kampen diagram for each of $w$ 's cyclic conjugates. We use induction on $L_{w}$ to prove that $\Delta_{\mathscr{P}}(w) \leq h\left(|w|_{\mathscr{P}}+L_{w}\right)$ if $L_{w}>1$ and $\Delta_{\mathscr{P}}(w) \leq h\left(|w|_{\mathscr{P}}\right)$ if $L_{w}=1$. Since $L_{w} \leq|w|_{\mathscr{P}}$, this would yield the fact that $\Delta_{\mathscr{P}}(w) \leq h\left(2 \cdot|w|_{\mathscr{P}}\right)$.

If $L_{w}=1$, then as in the proof of Proposition 3.1, $w$ can be thought of as a word in either $\mathscr{P}_{A}$ or $\mathscr{P}_{B}$ and we have $\Delta_{\mathscr{P}}(w) \leq h\left(|w|_{\mathscr{P}}\right)$.

Suppose that the result is true for words of cyclic free product length less than $L_{w}$. Since $w$ represents the identity in $A *_{C} B$ at least one of the subwords, say $w_{i}$, represents an element of $C$, say $c_{j}$. Now $w_{i} c_{j}^{-1}$ can be thought of as a word in one of the subpresentations $\mathscr{P}_{A}$ or $\mathscr{P}_{B}$ representing the identity element. So there is a Van Kampen diagram $D_{i}$ for $w_{i} c_{j}^{-1}$ over $\mathscr{P}_{A}$ or $\mathscr{P}_{B}-$ which is also a diagram over $\mathscr{P}$-having area bounded above by $h\left(\left|w_{i}\right|_{\mathscr{P}}+1\right)$.

Consider the word $w^{\prime}$ gotten from $w$ by replacing $w_{i}$ with $c_{j}$. We have two cases, depending on whether $L_{w}=2$ or $L_{w}>2$. In both cases $\left|w^{\prime}\right|_{\mathscr{P}}=$ $|w|_{\mathscr{P}}-\left|w_{i}\right|_{\mathscr{P}}+1$.

Suppose $L_{w}=2$. Then $L_{w^{\prime}}=1$ and it follows that $\Delta_{\mathscr{P}}\left(w^{\prime}\right) \leq h\left(\left|w^{\prime}\right|_{\mathscr{P}}\right)$. Let $D^{\prime}$ be a Van Kampen diagram for $w^{\prime}$ with area bounded above by $h\left(\left|w^{\prime}\right|_{\mathscr{P}}\right)$. Glue $D^{\prime}$ and $D_{i}$ together along the edge mapping to $c_{j}$ (see Figure 3.2). This yields a Van Kampen diagram for $w$ having area bounded above by $h\left(\left|w^{\prime}\right|_{\mathscr{P}}\right)+$ $h\left(\left|w_{i}\right|_{\mathscr{P}}+1\right)$ which by subnegativity is

$$
\leq h\left(\left|w^{\prime}\right|_{\mathscr{P}}+\left|w_{i}\right|_{\mathscr{P}}+1\right)=h\left(|w|_{\mathscr{P}}+2\right)=h\left(|w|_{\mathscr{P}}+L_{w}\right)
$$

as claimed.

The argument when $L_{w}>2$ is similar; only the arithmetic changes. In this case $L_{w^{\prime}}=L_{w}-2$ and by induction

$$
\Delta_{\mathscr{P}}\left(w^{\prime}\right) \leq h\left(\left|w^{\prime}\right|_{\mathscr{P}}+L_{w^{\prime}}\right)=h\left(\left|w^{\prime}\right|_{\mathscr{P}}+L_{w}-2\right) .
$$

Let $D^{\prime}$ be a diagram for $w^{\prime}$ with area bounded above by $h\left(\left|w^{\prime}\right|_{\mathscr{P}}+L_{w}-2\right)$. Again, glue $D^{\prime}$ and $D_{i}$ together along the edge mapping to $c_{j}$. This yields a diagram for $w$ having area bounded above by $h\left(\left|w^{\prime}\right|_{\mathscr{P}}+L_{w}-2\right)+h\left(\left|w_{i}\right|_{\mathscr{P}}+1\right)$ which by subnegativity is

$$
\leq h\left(\left|w^{\prime}\right|_{\mathscr{P}}+\left|w_{i}\right|_{\mathscr{P}}+L_{w}-1\right)=h\left(|w|_{\mathscr{P}}+L_{w}\right)
$$

as desired.

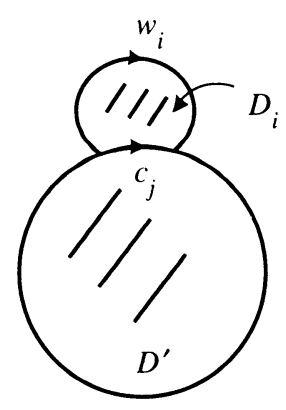

FIGURE 3.2 
So $\Delta_{\mathscr{P}}(w) \leq h\left(2 \cdot|w|_{\mathscr{P}}\right)$. Taking the maximum over $N_{\mathscr{P}}(l)$ finishes the proof.

As mentioned above, when the factors $A$ and $B$ are negatively curved the preceding yields Gromov's result, i.e. the resulting group $G$ is negatively curved. For example, we can conclude that $S L_{2}(\mathbf{Z})$ is negatively curved.

The above proof easily adapts to the case of an HNN extension, yielding the following result:

Proposition 3.3. Suppose $A$ is finitely presented and $G=A{ }^{*_{C}} \phi$ is an $H N N$ extension of $A$, where $C$ is finite. Then $\delta_{G} \preccurlyeq \bar{\delta}_{A}$.

Proof. The proof is similar to that of Proposition 3.2 above. The main difference is that the cyclic free product length is replaced by the number of occurrences of the stable letter.

As above, enumerate $C$ by $1, c_{1}, \ldots, c_{d}$. Also enumerate $C^{\prime}=\phi(C)$ by $1, c_{1}^{\prime}, \ldots, c_{d}^{\prime}$ where $\phi\left(c_{i}\right)=c_{i}^{\prime}$ for each $i$. Choose

$$
\mathscr{P}_{A}=\left\langle x_{1}, \ldots, x_{n}, c_{1}, \ldots, c_{d}, c_{1}^{\prime}, \ldots, c_{d}^{\prime} \mid r_{1} \ldots, r_{u}\right\rangle \text {, }
$$

a finite presentation of $A$. Then

$$
\mathscr{P}=\left\langle x_{i}, c_{j}, t \mid r_{s}, t c_{j} t^{-1}=c_{j}^{\prime}\right\rangle
$$

is a finite presentation for $G$.

Given $w \in N_{\mathscr{P}}(l)$ we write $w=w_{0} t^{\varepsilon_{1}} w_{1} t^{\varepsilon_{2}} \cdots t^{\varepsilon_{H}} w_{H}$ where $H \geq 0$, each $\varepsilon_{i} \in\{-1,+1\}$, and each subword $w_{i}$ is a word in $\mathscr{P}_{A}$-though possibly the null word. Call $H$ the $H N N$ length of $w$. We will prove by induction on $H$ that $\Delta_{\mathscr{P}}(w) \leq \bar{\delta}_{A}\left(|w|_{\mathscr{P}}\right)+(H / 2)$.

If $H=0$ then $w$ is a word in $\mathscr{P}_{A}$ and so $\Delta \mathscr{P}(w) \leq \delta_{\mathscr{P}}\left(|w|_{\mathscr{P}}\right) \leq \bar{\delta}_{\mathscr{P}}\left(|w|_{\mathscr{P}}\right)$.

If $H>0$ then as $w$ represents the identity element in $G$ we must have $H>1$. Moreover, there is some $i$ with $1 \leq i<H$ and with $t^{\varepsilon_{i}} w_{i} t^{\varepsilon_{i+1}}$ being of either the form $t w_{i} t^{-1}$ with $w_{i}$ representing an element, say $c_{j}$, of $C$ in $A$ or the form $t^{-1} w_{i} t$ with $w_{i}$ representing an element, say $c_{j}^{\prime}$, of $C^{\prime}$ in $A$. Let $y$ denote $c_{j}$ or $c_{j}^{\prime}$, as the case may be. Then $w_{i} y^{-1}$ represents the identity element in $A$. Choose a Van Kampen diagram $D_{i}$ over $\mathscr{P}_{A}$, and thus also over $\mathscr{P}$, for $w_{i} y^{-1}$ with area bounded above by $\delta_{\mathscr{P}_{A}}\left(\left|w_{i}\right|_{\mathscr{P}}+1\right)$ which is necessarily $\leq \bar{\delta}_{\mathscr{P}_{A}}\left(\left|w_{i}\right|_{\mathscr{P}}+1\right)$. Replace $w_{i}$ be $y$. The resulting word $w^{\prime}$ has a subword of the form $t c_{j} t^{-1}$ or $t^{-1} c_{j}^{\prime} t$. So we can use a diagram $D^{\prime}$ consisting of a single two-cell to change $w^{\prime}$ to a word $w^{\prime \prime}$ with HNN length being $H-2$ (see Figure 3.3) and with

$$
\left|w^{\prime \prime}\right|_{\mathscr{P}}=|w|_{\mathscr{P}}-\left(\left|w_{i}\right|_{\mathscr{P}}+2\right)+1 \text {. }
$$

So by induction, there is a diagram $D^{\prime \prime}$ for $w^{\prime \prime}$ having area bounded above

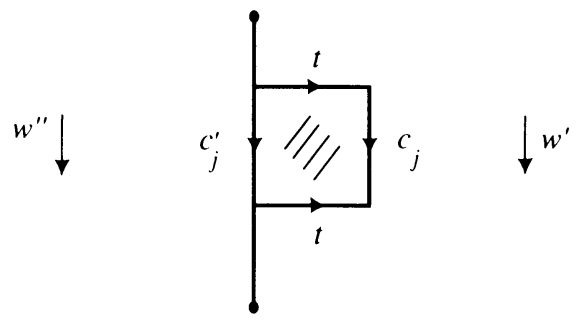

FIGURE 3.3 


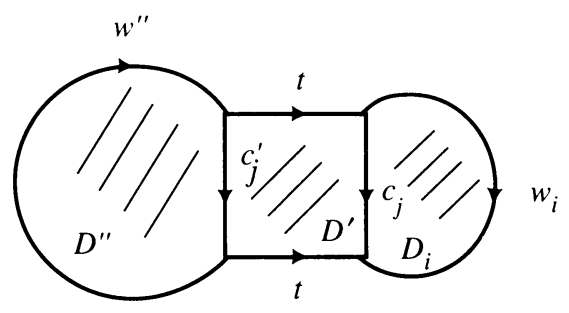

FIGURE 3.4

by $\delta_{\mathscr{P}_{A}}^{-}\left(\left|w^{\prime \prime}\right| \mathscr{P}\right)+(H-2) / 2$. Now glue together the diagrams $D_{i}, D^{\prime}$ and $D^{\prime \prime}$ as indicated in Figure 3.4, obtaining a Van Kampen diagram for $w$ with area bounded above by

$$
\bar{\delta}_{\mathscr{P}_{A}}\left(\left|w_{i}\right|_{\mathscr{P}}+1\right)+1+\bar{\delta}_{\mathscr{P}_{A}}\left(\left|w^{\prime \prime}\right| \mathscr{P}\right)+(H-2) / 2
$$

which by subnegativity is

$$
\leq \bar{\delta}_{\mathscr{P}_{A}}\left(\left|w_{i}\right|_{\mathscr{P}}+1+\left|w^{\prime \prime}\right| \mathscr{P}\right)+(H / 2)=\bar{\delta}_{\mathscr{P}_{A}}\left(|w|_{\mathscr{P}}\right)+(H / 2)
$$

as claimed.

Now $H \leq|w|_{\mathscr{P}}$, so $\Delta_{\mathscr{P}}(w) \leq \bar{\delta}_{\mathscr{P}_{A}}\left(|w|_{\mathscr{P}}\right)+\left(|w|_{\mathscr{P}} / 2\right)$, and taking the maximum over $N_{\mathscr{P}}(l)$ yields the desired $\delta_{\mathscr{P}} \preccurlyeq \bar{\delta}_{\mathscr{P}_{A}}$.

Since we can use the subnegative closure to compute the degree, we obtain the following corollary.

Corollary 3.4. Let $A$ and $B$ be finitely presented groups. Suppose $C$ is finite and $\phi: C \approx \phi(C)$. Then

(1) $\operatorname{deg}(A * B)=\max (\operatorname{deg}(A), \operatorname{deg}(B))$,

(2) $\operatorname{deg}\left(A *_{C} B\right) \leq \max (\operatorname{deg}(A), \operatorname{deg}(B))$,

(3) $\operatorname{deg}\left(A *_{C} \phi\right) \leq \operatorname{deg}(A)$.

We now turn to a class of amalgamations that may possibly increase the degree by one. We start with a few definitions.

If $G$ is a group, $X$ is a set of generators, and $g \in G$, write $l_{X}(g)$ for the shortest length of words in $X \cup X^{-1}$ representing $g$.

Given a pair of finitely generated groups $S \subset G$, we say that $S$ is isometric in $G$ if there are generating sets $X_{S}$ and $X_{G}$ for $S$ and $G$ respectively with $X_{S} \subset X_{G}$ and with $l_{X_{S}}(u)=l_{X_{G}}(u)$ for all $u \in S$ (since $X_{S} \subset X_{G}$ we always have $\left.l_{X_{G}}(u) \leq l_{X_{S}}(u)\right)$. In other words, the inclusion map induces an isometric embedding of the associated Cayley graphs.

Following Gromov, we say that $S$ is plus-isometric in $G$ if, for some choice of generating sets, there is a constant $k$ so that $l_{X_{S}}(u) \leq l_{X_{G}}(u)+k$ for all $u \in S$. So there is a plus-isometric embedding of the associated Cayley graphs.

An amalgam $A *_{S} B$, is called plus-isometric if $S$ is finitely generated and is simultaneously plus-isometric in both $A$ and $B$. In other words, there are finite generating sets $X_{S}$ for $S, X_{A}$ for $A$, and $X_{B}$ for $B$ with $X_{S} \subset X_{A}$ and $X_{S} \subset X_{B}$, and there is a constant $k$ such that $l_{X_{S}}(u) \leq l_{X_{A}}(u)+k$ and $l_{X_{S}}(u) \leq l_{X_{B}}(u)+k$ for all $u \in S$. Here we have a single Cayley graph $\Gamma\left(S, X_{S}\right)$ for $S$ and two plus-isometric embeddings, one into $\Gamma\left(A, X_{A}\right)$ and the other into $\Gamma\left(B, X_{B}\right)$. We will also call the generating sets plus-isometric. 
By using induction on the cyclic free product length we prove our next result on the Dehn function of plus-isometric amalgams. Strangely enough, we do not need to take subnegative closures. We are grateful to J. Alonso for pointing out that our argument for isometric amalgams actually applies to the plus-isometric case.

Proposition 3.5. Suppose $A$ and $B$ are finitely presented and $G=A{ }_{S} B$ is $a$ plus-isometric amalgam. Then

$$
\delta_{G} \preccurlyeq(\text { a linear function }) \cdot \max \left(\delta_{A}, \delta_{B}\right) .
$$

Proof. Choose a finite presentation for $A$ of the form

$$
\mathscr{P}_{A}=\left\langle x_{1}, x_{2}, \ldots, x_{n}, s_{1}, s_{2}, \ldots, s_{d} \mid r_{1}, r_{2}, \ldots, r_{u}\right\rangle
$$

and a finite presentation for $B$ of the form

$$
\mathscr{P}_{B}=\left\langle y_{1}, y_{2}, \ldots, y_{m}, s_{1}, s_{2}, \ldots, s_{d} \mid v_{1}, v_{2}, \ldots, v_{t}\right\rangle,
$$

where $\left\{s_{z}\right\},\left\{x_{i}, s_{z}\right\}$, and $\left\{y_{j}, s_{z}\right\}$ are plus-isometric generating sets for $S$, $A$, and $B$ respectively. Let $k$ be the constant given in the definition of plusisometry. Take, as presentation of $A *_{S} B$,

$$
\mathscr{P}=\left\langle x_{i}, y_{j}, s_{z} \mid r_{p}, v_{q}\right\rangle \text {. }
$$

Given a word $w$ in $\mathscr{P}$, let $L_{w}$ denote its cyclic free product length. Set

$$
h(l)=\max \left(\delta_{\mathscr{P}_{A}}(l), \delta_{\mathscr{P}_{B}}(l)\right) .
$$

We will prove by induction on $L_{w}$ that for $w \in N_{\mathscr{P}}$ we have $\Delta_{\mathscr{P}}(w) \leq\left(L_{w}\right)$. $h\left(2|w|_{\mathscr{P}}+k L_{w}\right)$.

Suppose $L_{w}=1$, then, as we have seen above, $w$ can be viewed as a word over either $\mathscr{P}_{A}$ or $\mathscr{P}_{B}$ and we can conclude that $\Delta_{\mathscr{P}}(w) \leq h\left(|w|_{\mathscr{P}}\right)$, which is $\leq h\left(2|w|_{\mathscr{P}}+k\right)$ because $h$ is monotone increasing.

Assume the result is true for any word of cyclic free product length less than $L_{w}$. As usual, write $w$ as a product of $L_{w}$ subwords $w_{i}$ which alternate between involving some $x_{i}$ and some $y_{j}$. Since $w$ represents the identity element, there is some subword, say $w_{i}$, that represents an element of $S$ in either $\mathscr{P}_{A}$ or $\mathscr{P}_{B}$, say $\mathscr{P}_{A}$. Since we have a plus-isometric amalgam, we may find a word $u$ in the generators for $S$ of length bounded by $\left|w_{i}\right|_{\mathscr{P}}+k$. Then the word $w_{i} u^{-1}$ represents the trivial element in $A$ and $\mathrm{s}$ there is a Van Kampen diagram $D_{i}$ over $\mathscr{P}_{A}$-and have over $\mathscr{P}$-for it with area bounded above by

$$
\delta_{\mathscr{P}}\left(\left|w_{i} u^{-1}\right|_{\mathscr{P}}\right) \leq h\left(2\left|w_{i}\right|_{\mathscr{P}}+k\right) \leq h\left(2|w|_{\mathscr{P}}+k\right) .
$$

Consider the word $w^{\prime}$ obtained from $w$ by replacing $w_{i}$ with $u$. We have $\left|w^{\prime}\right|_{\mathscr{P}} \leq|w|_{\mathscr{P}}+k$ and $L_{w^{\prime}}=L_{w}-2$. By induction (and the fact that $h$ is monotone) there is a Van Kampen diagram $D^{\prime}$ for $w^{\prime}$ having area bounded above by

$$
\left(L_{w}-2\right) \cdot h\left(2\left(|w|_{\mathscr{P}}+k\right)+k\left(L_{w}-2\right)\right)=\left(L_{w}-2\right) \cdot h\left(2|w|_{\mathscr{P}}+k L_{w}\right) .
$$

Now glue $D_{i}$ and $D^{\prime}$ together along the subword mapping to $u$ (the picture, except for the labels, is the same as that in Figure 3.2) obtaining a Van Kampen diagram for $w$ with area bounded above by

$$
h\left(2|w|_{\mathscr{P}}+k\right)+\left(L_{w}-2\right) \cdot h\left(2|w|_{\mathscr{P}}+k L_{w}\right) .
$$


This is clearly less than $L_{w} \cdot h\left(2|w|_{\mathscr{P}}+k L_{w}\right)$, completing the induction.

So we have

$$
\Delta_{\mathscr{P}}(w) \leq L_{w} \cdot h\left(2|w|_{\mathscr{P}}+k L_{w}\right) \leq|w|_{\mathscr{P}} \cdot h\left((2+k)|w|_{\mathscr{P}}\right)
$$

because $L_{w} \leq|w|_{\mathscr{P}}$. Taking the maximum over $N_{\mathscr{P}}(l)$ proves that $\delta_{\mathscr{P}}(l) \preccurlyeq$ $l \cdot \max \left(\delta_{\mathscr{P}_{A}}(l), \delta_{\mathscr{P}_{B}}(l)\right)$ as desired.

The preceding may be applied to show that the group of the trefoil knot satisfies a quadratic isoperimetric inequality.

We end with an immediate corollary.

Corollary 3.7. Suppose $A$ and $B$ are finitely presented. If $G=A{ }^{*} C B$ is plusisometric then $\operatorname{deg}(G) \leq \max (\operatorname{deg}(A), \operatorname{deg}(B))+1$.

\section{REFERENCES}

[A] J. M. Alonso, Inegalités isopérimétriques et quasi-isométries, C. R. Acad. Sci. Paris 311 (1990), 761-764.

[Ba et al.] W. Ballman, E. Ghys, A. Haefliger, P. de la Harpe, E. Salem, R. Strebel, and M. Troyanov, Sur les groupes hyperboliques d'après Mikhael Gromov, Birkhäuser, 1990.

[BGSS] G. Baumslag, S. Gersten, M. Shapiro, and H. Short, Automatic groups and amalgams, J. Pure Appl. Algebra 76 (1991), 229-316.

[Br] S. G. Brick, Dehn functions of groups and extensions of complexes, Preprint.

[CDP] M. Coornaert, T. Delzant, and A. Papadopoulos, Notes sur les groupes hyperboliques de Gromov, Lecture Notes in Math., vol. 1441, Springer, 1990.

[CEHLPT] J. W. Cannon, D. B. A. Epstein, D. F. Holt, S. V. F. Levy, M. S. Paterson, and W. P. Thurston, Word processing and group theory, Jones and Bartlett, 1992.

[Ge] S. M. Gersten, Dehn functions and $l_{1}$-norms of finite presentations, Algorithms and Classifications in Combinatorial Group Theory (G. Baumslag and C. F. Miller III, eds.), Math. Sci. Res., Springer-Verlag, 1991.

[Gr] M. Gromov, Hyperbolic groups, Essays in Group Theory (S. M. Gersten, ed.), Math. Sci. Res., Springer-Verlag, 1987.

[Sh] H. Short, Regular subgroups of automatic groups, Preprint.

Department of Mathematics, University of Oklahoma, Norman, Oklahoma 73019 Current address: Department of Mathematics, California State University at Fresno, Fresno, California 93740

E-mail address: stephenb@zimmer.csufresno.edu 